\title{
CONVÍVIO DE ANIMAIS DE ESTIMAÇÃO ENTRE IDOSOS: UM ESTUDO DE BASE POPULACIONAL NO SUL DO BRASIL
}

\author{
Living with pets among the elderly: a population-based study in southern Brazil
}

https://doi.org/10.18593/eba.24966

Recebido em 1 de agosto de 2020 | Aceito em 1 de dezembro de 2020

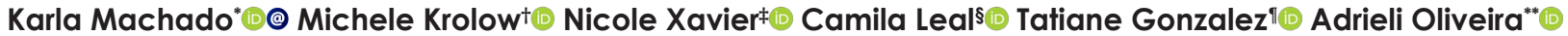

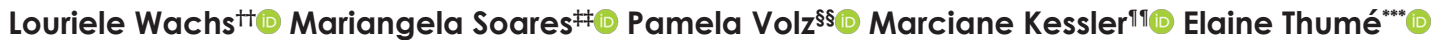

\footnotetext{
Mestre em Saúde Pública pela Universidade Federal do Rio Grande; Graduada em Nutrição pela Universidade Federal de Pelotas; Professora na Universidade Federal de Pelotas.

Graduada em Enfermagem pela Universidade Federal de Pelotas.

‡ Graduanda em Enfermagem pela Universidade Federal de Pelotas.

$\S$ Graduada em Enfermagem pela Universidade Federal de Pelotas.

" Mestre em Ciências da Saúde pela Universidade Federal do Rio Grande; Graduada em Nutrição pela Universidade Federal de Pelotas; Professora na Universidade Federal do Rio Grande.

** Graduanda em Enfermagem pela Universidade Federal de Pelotas.

†† Doutora e Mestre em Ciências da Saúde pela Universidade Federal de Pelotas; Professora na Universidade Federal de Pelotas.

执 Doutora e Mestre em Ciências da Saúde pela Universidade Federal de Pelotas; Professora na Universidade Federal de Pelotas.

$\S \S$ Doutora e Mestre em Ciências da Saúde pela Universidade Federal de Pelotas; Professora na Universidade Federal de Pelotas.

ก" Doutora e Mestre em Ciências da Saúde pela Universidade Federal de Pelotas; Professora na Universidade Federal de Pelotas.

*** Doutora em Epidemiologia pela Universidade Federal de Santa Catarina; Mestre em Enfermagem pela Universidade Federal de Santa Catarina; Professora na Universidade Federal de Pelotas.
}

Resumo: Durante o envelhecimentoa convivência comanimais deestimação podeajudara pessoa idosa a atravessarsituações difíceis, tornando os animais de estimação um suporte social. O objetivo do estudo foi conhecer a prevalência de idosos em convívio com animais de estimação e sua associação com características sociodemograficas e de saúde. Estudo transversal de base populacional. A coleta de dados ocorreu por meio de inquérito domiciliar com idosos de 60 anos ou mais, residentes na área urbana do município de Bagé, RS, no ano de 2008. A variável dependente foi convivência com animais de estimação e as independentes incluíram características sociodemograficas e de saúde. Foram realizadas análises descritivas e bivariadas, para verificar as associações foi utilizado o teste de exato de Fisher para heterogeneidade. O nível de significância estatístico utilizado foi de $5 \%$ para testes bicaudais. Todas análises foram realizadas no programa Stata versão 14.o. A amostra foi de 1.593 idosos. A prevalência geral de convívio com animais de estimação foi de (69,0\%; IC 95\%: 66,6; 71,2). As variáveis associadas com o desfecho foram: sexo masculino (72\%), menor faixa etária (6o a 74 anos - 72,9\%), viver com companheiro (72,9\%), não morar sozinho, não receber aposentadoria $(72,9 \%)$ e não ter referido queda no último ano (70,7\%). Encontrou-se uma elevada prevalência de idosos em convívio com animais de estimação, contudo mais estudos são necessários para melhor compreender como a convivência com os animais se reflete em melhorias de saúde para a população idosa.

Palavras-chave: Idoso. Vínculo Homem-Animal de Estimação. Animais domésticos. Saúde do idoso. Estudos transversais.

@Autor correspondente: Mestre em Saúde Pública pela Universidade Federal do Rio Grande; Graduada em Nutrição pela Universidade Federal de Pelotas; Professora na Universidade Federal de Pelotas, Rua Gomes Carneiro, 1, Centro, 96010-610, Pelotas, Rio Grande do Sul, Brasil; https://orcid.org/0000-0003-1765-1435; karlamachadok@gmail.com 
Abstract: During aging, living with pets can help the elderly to go through difficult situations, making pets a social support. The objective of this study was to know the prevalence of elderly people living with pets and its association with sociodemographic and health characteristics. Methods: Cross-sectional population-based study. Data collection took place through a household survey with elderly people aged 6o or over, living in the urban area of the municipality of Bagé, RS, in 20o8. The dependent variable was living with pets and the independent ones included sociodemographic and health characteristics. Descriptive and bivariate analyzes were performed and, to verify associations, Fisher's exact test for heterogeneity was used. The level of statistical significance used was 5\% for twotailed tests. All analyzes were performed using the Stata version 14.o program. The sample consisted of 1,593 elderly people. The general prevalence of living with pets was (69.0\%; 95\% CI: 66.6; 71.2). The variables associated with the outcome were: male gender (72.0\%), younger age group (6o to 74 years - 72.9\%), living with a partner (72.9\%), not living alone, not receiving retirement (72.9\%) and not having reported a drop in the last year (70.7\%). There was a high prevalence of elderly people living with pets, however more studies are needed to better understand how living with animals is reflected in health improvements for the elderly population.

Keywords: Aged. Bonding Human-Pet. Animals domestic. Health of the elderly. Cross-sectional studies.

\section{INTRODUÇÃO}

São diversas as particularidades que pautam a dinâmica do envelhecimento e suas dimensões. As situações vivenciadas e escolhas que são feitas ao longo da vida acabam por, muitas vezes, individualizar o processo de envelhecimento. ${ }^{1}$ Nesse sentido, seja por hábitos remanescentes ao longo da vida ou por ordem fisiológica, o processo de envelhecimento possibilita o surgimento ou agravamento de doenças crônicas que afetam a qualidade de vida do idoso. No entanto, não é só a doença em si que compromete a condição do bem viver da pessoa, o desgaste físico também pode contribuir com danos psíquicos, como a depressão e o estresse, podendo afetar a vida social do idoso. ${ }^{2,3}$

Assim, durante o envelhecimento, a posse e o convívio entre idosos e animais de estimação tornam-se especialmente importantes. A convivência com esses animais pode ajudar a pessoa idosa a atravessar situações difíceis, pois é nessa fase da vida que se vive a ausência de parentes, a perda de entes queridos, o distanciamento dos colegas de trabalho, a alteração dos papéis sociais e a aceitação da própria velhice, como isso os animais de estimação tornando-se um suporte social.4,5 Além de companhia, esses também estimulam a responsabilidade, promovem o bem-estar, com melhoria na qualidade de vida das pessoas, e contribuem nos aspectos biopsicossociais, como diminuição do estresse, diminuição da dor e da ansiedade, aumento de endorfina e melhoria no relacionamento interpessoal. . $^{-9}$

O convívio com um animal de estimação pode trazer inúmeros benefícios a saúde do idoso, dentre eles o alívio da rotina, momentos de lazer, companhia, redução da ansiedade, demonstração de afeto, momentos de relaxamento e alegria. ${ }^{10}$ Ainda, motiva a sociabilidade e promove o incentivo à realização de atividades físicas. Esses fatores certamente contribuem com a qualidade de vida da pessoa idosa, que muitas vezes convive com características fisiológicas procedentes do processo de envelhecimento, representando melhorias na saúde, fomentando a participação e bem-estar na comunidade."

Apesar da importância de convívio doméstico de animais com idosos e dos benefícios que essa convivência propicia, esse ainda é um assunto pouco abordado na literatura, portanto, o objetivo do estudo foi conhecer a prevalência de idosos em convívio com animais de estimação e sua associação com características sociodemograficas e de saúde no município de Bagé, Rio Grande do Sul. 


\section{MATERIAL E MÉTODOS}

Trata-se de um estudo transversal de base populacional, realizado com indivíduos de 60 anos ou mais, residentes na área de abrangência dos serviços de atenção básica à saúde da zona urbana de Bagé, município situado no interior do Rio Grande do Sul. Em 2008, ano da coleta de dados, o município possuía aproximadamente 115.755 habitantes, ${ }^{12}$ deste total $82,0 \%$ viviam na zona urbana. No mesmo ano, havia 15 Unidades de Saúde da Família (USF), totalizando 19 equipes, responsáveis por $51,0 \%$ da população urbana do município e cinco unidades mantinham o modelo tradicional de atenção básica e atendiam o restante da população.

O tamanho de amostra foi calculado considerando $10 \%$ de perdas e recusas, um efeito de delineamento de $1.3,80 \%$ de poder para detectar riscos relativos de 1.5 de exposições que afetavam, no mínimo, $4 \%$ da população. Para garantir a representatividade da amostra, o processo de amostragem foi realizado em múltiplos estágios. Inicialmente, a amostra foi localizada a partir da área de abrangência de cada uma das Unidades Básicas de Saúde (UBS) e, posteriormente, dividida em microáreas, com identificação numérica de cada quadra, sorteando aleatoriamente o ponto inicial de coleta de dados. Com o intuito de garantir que todos os domicílios tivessem a mesma probabilidade de compor a amostra, foi empregado o pulo sistemático de uma a cada cinco residências. Este procedimento garantiu que fossem amostrados quatro em cada cinco domicílios. Todos os moradores com 60 anos ou mais foram convidados a participar do estudo e incluídos mediante leitura e assinatura do Termo de Consentimento Livre e Esclarecido (TCLE).

A coleta de dados foi realizada de julho a novembro de 2008 , por nove entrevistadoras previamente treinadas. As entrevistas foram feitas no domicílio selecionado, com todos os moradores elegíveis, por meio de um questionário estruturado, previamente testado, com questões pré-codificadas. Foram consideradas perdas/ recusas as entrevistas não realizadas após três tentativas em dias e horários diferentes. E, foram excluídos os indivíduos que, no momento da entrevista, estavam viajando, privados de liberdade por decisão judicial ou residindo em instituição de longa permanência. No caso de pessoas sem condições físicas ou mentais para responder o questionário, este foi aplicado ao cuidador principal.

O desfecho do estudo, convívio com os animais foi investigado por meio da questão " $O$ (a) Sr. (a) tem algum animal de estimação em sua casa?" - com opção de resposta dicotômica (não/ sim), em caso afirmativo era questionado qual animal de estimação o entrevistado possuía.

O contexto demográfico e socioeconômico foi analisado por meio das seguintes variáveis independentes: sexo (masculino; feminino), idade (6o a 74; 75 anos ou mais), cor da pele (branca; preta/amarela/parda/indígena), situação conjugal (casado ou com companheiro; viúvo; solteiro ou separado), escolaridade - em anos completos de estudo (nenhum; um a sete; e oito ou mais), classificação econômica - ANEP (A/B; C; D/E), aposentadoria (não; sim), morar sozinho (não; sim). As variáveis de saúde utilizadas foram: autopercepção de saúde (ótima/boa; regular; ruim péssima), presença de sintomas de depressão avaliada através da Escala de Depressão Geriátrica, ${ }^{13}$ com os resultados divididos em depressão presente (de o a 5) e ausente ( $\geq 6$ ) e história de queda no último ano (não; sim).

Para a análise dos dados, foi utilizado o programa Stata ${ }^{\circledR}$ versão 14.0 (StataCorp/ 
CollegeStation, Estados Unidos). Foi utilizada a estatística descritiva com o cálculo de distribuição proporcional. Para avaliar a diferença estatística entre os grupos foi empregado teste de exato de fisher para heterogeneidade ( $\mathrm{p}$-valor $<0,05$ ).

\subsection{APROVAÇÃO ÉTICA}

O projeto foi aprovado no Comitê de Ética em Pesquisa da Faculdade de Medicina da UFPel, através do ofício n⿳o. 015/o8. Os princípios éticos foram respeitados, a partir da apresentação aos entrevistados do Termo de Consentimento Livre e Esclarecido, assinado por todos, de maneira a garantir o direito de não participação na pesquisa e o anonimato dos participantes.

\section{RESULTADOS}

Foi localizado um total de 1.713 idosos elegíveis e 1.593 foram entrevistados. A proporção de resposta foi de $93 \%$, com $4 \%$ de perdas e $3 \%$ de recusas. A maioria dos entrevistados era do sexo feminino $(62,8 \%)$, a cor da pele branca foi predominante $(78,6 \%)$, a média de idade foi de 71,2 anos $(\mathrm{DP} \pm 8,2)$ e os idosos viúvos correspondiam a 33,8\% da amostra. Referente à classificação socioeconômica, 27,1\% dos entrevistados pertencia à classe $\mathrm{D} / \mathrm{E}$, quase um quarto da amostra não possuía nenhum ano de estudo completo $(23,7 \%)$ e aproximadamente três quartos $(71,7 \%)$ estavam aposentados. Quanto a autopercepção de saúde, $58,8 \%$ consideraram seu estado de saúde bom ou muito bom, 14,9\% de idosos tiveram rastreio para depressão e $28,0 \%$ relataram queda no último ano (Tabela 1).
Tabela 1 - Distribuição proporcional das características sociodemográficas e de saúde na amostra. Bagé, RS, 2008 ( $\mathrm{N=1.593).}$

\begin{tabular}{lll}
\hline Variáveis & $\mathrm{N}$ & $\%$ \\
\hline Sexo & & \\
\hline Masculino & 593 & 37,2 \\
\hline Feminino & 1.000 & 62,8 \\
\hline Idade & & \\
\hline 60 a 74 anos & 1.096 & 68,8 \\
\hline 75 anos ou mais & 497 & 31,2 \\
\hline Cor da pele & & \\
\hline Branca & 1.252 & 78,6 \\
\hline Preta/ parda/ amarela/ indígena & 341 & 21,4 \\
\hline Situação Conjugal (n=1.592) & & \\
\hline Casado(a) ou com companheiro & 816 & 51,3 \\
\hline Solteiro(a) ou separado(a) & 238 & 14,9 \\
\hline Viúvo(a) & 538 & 33,8 \\
\hline Classificação Econômica (ANEP) & & \\
(n=1.581) & & \\
\hline A/B & 537 & 34,0 \\
\hline C & 615 & 38,9 \\
\hline D/E & \multicolumn{2}{|l}{27,1} \\
\hline Anos & & \\
\hline
\end{tabular}

Anos de estudo (em anos completos)

$(\mathrm{n}=1.572)$

\begin{tabular}{lll}
\hline Nenhum & 342 & 21,8 \\
\hline 1 a 7 & 858 & 54,6 \\
\hline$\geq 8$ & 372 & 23,6 \\
\hline
\end{tabular}

Aposentadoria

\begin{tabular}{lll}
\hline Não & 451 & 28,3 \\
\hline Sim & 1.142 & 71,7
\end{tabular}

Morar Sozinho

\begin{tabular}{lll}
\hline Não & 1.313 & 82,4 \\
\hline Sim & 280 & 17,6 \\
\hline
\end{tabular}

Autopercepção de Saúde ( $\mathrm{n}=1.540)$

\begin{tabular}{lll}
\hline Péssima/ruim & 109 & 7,1 \\
\hline Regular & 525 & 34,1 \\
\hline Boa/ótima & 906 & 58,8
\end{tabular}

Depressão $(\mathrm{n}=1.512)$

\begin{tabular}{lcc}
\hline Não & 1.240 & 82,0 \\
\hline Sim & 272 & 18,0 \\
\hline Queda no último ano $(\mathrm{n}=1.591)$ & & \\
\hline Não & 1.145 & 72,0 \\
\hline Sim & 446 & 28,0 \\
\hline Nota: & $\begin{array}{c}\text { N=frequência absoluta; } \\
\text { ANEP=frequência }\end{array}$ & relativa.
\end{tabular}


O convívio com os animais de estimação foi referido pela maioria dos idosos $(69,0 \%$; IC 95\%: 66,6; 71,2), destes $87,7 \%$ referiram ter cachorro como animal de estimação, 37,4 \% gato, $11,1 \%$ pássaro, $5,4 \%$ cavalo e $7,3 \%$ referiram outros animais (Figura 1).

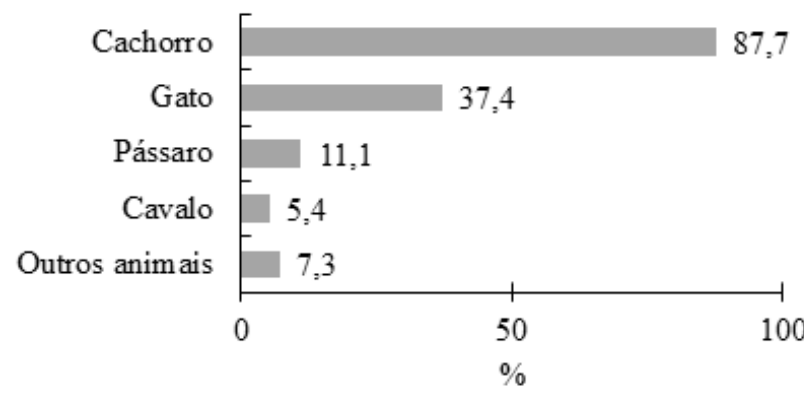

Figura 1 - Distribuição proporcional do tipo de animal de estimação entre os idosos. Bagé, RS, 2008 ( $n=1.593)$.
As maiores proporções de idosos em convívio de animais de estimação foram observadas entre os idosos do sexo masculino (72,0\%), com idade de 6 o a 74 anos $(72,9 \%)$, casados ou que viviam com companheiro (72,9\%), que não recebiam aposentadoria $(72,9 \%)$, que não moravam sozinho $(70,9 \%)$, que não apresentavam rastreio para depressão $(70,3 \%)$ e por fim, nos idosos que não tinham sofrido queda o último ano (70,7\%) (Tabela 2).

Tabela 2 - Distribuição proporcional entre a população idosa em convívio com animais de estimação segundo características sociodemográficas e de saúde. Bagé, RS, 2008 ( $\mathrm{N=1.593).}$

\begin{tabular}{|c|c|c|c|c|c|}
\hline \multirow{3}{*}{ Variáveis } & \multicolumn{5}{|c|}{ Idosos em convívio com animais de estimação } \\
\hline & \multicolumn{2}{|c|}{ Não } & \multicolumn{2}{|c|}{ Sim } & \multirow[t]{2}{*}{ Valor $\mathbf{p}^{*}$} \\
\hline & $\mathbf{N}$ & $\%$ & $\mathbf{N}$ & $\%$ & \\
\hline Sexo & & & & & 0,050 \\
\hline Masculino & 166 & 28,0 & 426 & 72,0 & \\
\hline Feminino & 238 & 32,8 & 671 & 67,2 & \\
\hline Idade & & & & & 0,001 \\
\hline 60 a 74 anos & 297 & 27,1 & 799 & 72,9 & \\
\hline 75 anos ou mais & 197 & 39,8 & 298 & 60,2 & \\
\hline Cor da pele & & & & & 0,129 \\
\hline Branca & 400 & 32,0 & 850 & 68,0 & \\
\hline Preta/ parda/ amarela/ indígena & 94 & 27,6 & 247 & 72,4 & \\
\hline Situação Conjugal $(\mathrm{n}=1.592)$ & & & & & 0,003 \\
\hline Casado (a) ou com companheiro & 221 & 27,1 & 593 & 72,9 & \\
\hline Solteiro (a) ou separado (a) & 84 & 35,3 & 154 & 64,7 & \\
\hline Viúvo (a) & 188 & 34,9 & 350 & 65,1 & \\
\hline Classificação Econômica (ANEP) $(\mathrm{n}=1.581)$ & & & & & 0,808 \\
\hline $\mathrm{A} / \mathrm{B}$ & 162 & 30,2 & 375 & 69,8 & \\
\hline $\mathrm{C}$ & 196 & 32,0 & 417 & 68,0 & \\
\hline $\mathrm{D} / \mathrm{E}$ & 134 & 31,2 & 295 & 68,8 & \\
\hline
\end{tabular}




\begin{tabular}{|c|c|c|c|c|c|}
\hline \multirow{3}{*}{ Variáveis } & \multicolumn{5}{|c|}{ Idosos em convívio com animais de estimação } \\
\hline & \multicolumn{2}{|c|}{ Não } & \multicolumn{2}{|c|}{ Sim } & \multirow[t]{2}{*}{ Valor $\mathbf{p}^{*}$} \\
\hline & $\mathbf{N}$ & $\%$ & $\mathbf{N}$ & $\%$ & \\
\hline Anos de estudo (em anos completos) $(\mathrm{n}=1.572)$ & & & & & 0,295 \\
\hline Nenhum & 116 & 31,3 & 255 & 68,7 & \\
\hline $1 \mathrm{a} 7$ & 256 & 29,9 & 601 & 70,1 & \\
\hline$\geq 8$ & 118 & 34,5 & 224 & 65,5 & \\
\hline Aposentadoria & & & & & 0,035 \\
\hline Não & 112 & 27,1 & 328 & 72,9 & \\
\hline Sim & 327 & 32,6 & 769 & 67,4 & \\
\hline Morar sozinho & & & & & $\leq 0,001$ \\
\hline Não & 382 & 29,1 & 929 & 70,9 & \\
\hline Sim & 112 & 40,0 & 168 & 60,0 & \\
\hline Autopercepção de Saúde (n=1.540) & & & & & 0,404 \\
\hline Péssima/ruim & 39 & 35,8 & 70 & 64,2 & \\
\hline Regular & 154 & 29,3 & 371 & 70,7 & \\
\hline Boa/ótima & 274 & 30,2 & 632 & 69,8 & \\
\hline Depressão $(n=1.512)$ & & & & & 0,070 \\
\hline Não & 368 & 29,7 & 872 & 70,3 & \\
\hline Sim & 96 & 35,3 & 176 & 64,7 & \\
\hline Queda no último ano $(\mathrm{n}=1.591)$ & & & & & 0,019 \\
\hline Não & 335 & 29,3 & 808 & 70,7 & \\
\hline Sim & 158 & 35,4 & 288 & 64,6 & \\
\hline
\end{tabular}

Nota: *teste exato de Fisher.

\section{DISCUSSÃO}

O presente estudo verificou elevada prevalência de idosos em convívio com animais de estimação. Entre esses, ser do sexo masculino, mais jovem ou de menor faixa etária, viver com companheiro, não morar sozinho, não receber aposentadoria e não ter referido queda no último ano foram as variáveis associadas ao convívio com animais de estimação.

O aumento da presença de animais de estimação nos lares brasileiros já foi relatado por outros autores. ${ }^{5,6,14}$ Ao longo dos anos os bichos de estimação conquistaram um espaço, sendo inclusive considerados membros da família. ${ }^{15}$ Muitos donos de animais destacam a pacificidade e a leveza desse convívio, pois esses trazem distração e são recíprocos nas demonstrações de afeto, bem como, estão disponíveis como companhia na maior parte do tempo. ${ }^{15}$

Heiden e Santos ${ }^{6}$ identificaram que mais de $70 \%$ dos idosos consideravam os animais de estimação como membros da família, prevalência similar ao nosso estudo. Da mesma maneira, estudo realizado em Paragominas, Pará, no ano de 2015, constatou que $81 \%$ dos entrevistados possuíam algum animal de estimação e que a proporção de animais por moradores no domicílio era de um para quatro. ${ }^{16}$ Uma informação que chama a atenção nos nossos achados é que além dos animais usualmente citados, como cachorro e gato, aparecem outros animais, dentre eles pássaro e cavalo. Uma possível explicação para tal achado pode ser atribuída às características do município, 
dentre elas a relevante atividade agropecuária desenvolvida, que predomina como principal fonte econômica da região e da própria cidade. Além disso, os limites entre as áreas urbana e rural são muito próximos, sendo mais comum a presença e posse de animais de estimação menos usuais.

Em nosso estudo encontramos uma maior prevalência de convívio com animais de estimação entre os idosos do grupo etário mais novo, supostamente o grupo mais ativo e independente. A menor posse entre os idosos mais velhos poderia ser decorrente de que com o avançar da idade alguns idosos necessitam de cuidados e apresentam maior grau de dependência e diminuição da autonomia. Além do que, para os cuidadores, o animal de estimação pode ser mais uma responsabilidade atribuída a eles. ${ }^{7,17}$

Na cidade de São Bento do Sul, Santa Catarina, um estudo realizado com idosos mostrou que aqueles que conviviam com animais domésticos apresentavam mudanças positivas no humor, tornando-os mais alegres e felizes. Ainda, $12 \%$ dos idosos relataram que após a chegada do animal o sentimento de solidão diminui. ${ }^{5}$ Para os idosos, ter um animal de estimação pode promover conforto em momentos complicados dessa fase da vida, além de melhorar a autoestima e a convivência social. ${ }^{14}$ Em nosso estudo os idosos que referiram conviver com animais de estimação tiveram as menores prevalências de depressão e referiram sua saúde como regular ou boa/ótima, porém essas associações não foram significativas, como apontadas em outros estudos. ${ }^{18}$ Diferenças metodológicas, como o tipo de instrumento e delineamento, podem justificar a falta de associação encontrada.

O convívio com animais não traz só benefícios para a saúde mental, mas também para a saúde física, pois os animais podem estimular a realização de exercícios, prática da mobilidade e esquecimento de dores físicas. ${ }^{10}$ Nosso estudo mostrou que os idosos com maior prevalência de posse de animais de estimação tiveram uma menor ocorrência de quedas. Por outro lado, alguns autores indicam que os animais podem estar ligados ao risco de quedas.,19 Estudo realizado em Pernambuco, com idosas frequentadoras do Núcleo de Atenção ao Idoso, mostrou uma associação entre o medo de se machucar ou cair e momentos de interação com os bichos. Porém, o convívio entre idosos e animais pode propiciar mais horas de lazer e atividade física, pois a presença de um animal de estimação pode trazer segurança e confiança para o idoso, desde que tomadas as devidas medidas de proteção. ${ }^{20}$

\section{Algumas limitações deste estudo} devem ser ponderadas. É possível que algumas associações estejam sujeitas à causalidade reversa, quando o desfecho modifica a exposição. Outro fator limitante é a ausência de análise ajustada, assim algumas associações encontradas na análise bruta podem ser fruto de fatores de confusão. Porém, a maior limitação do nosso estudo é a escassa quantidade de artigos publicados sobre a temática, o que certamente restringe e prejudica a comparação e discussão dos achados, principalmente os de caráter quantitativos como este.

Outra limitação do artigo é que se trata de dados coletados no ano de 2008, porém ao se comparar a população de idosos residentes no município no ano da coleta de dados e no ano de 2020, a proporção permanece estável, próxima a 14\%. Portanto, provavelmente, a prevalência do desfecho não variou muito nesse período, o que justifica a publicação do artigo. ${ }^{21}$ 
Como pontos positivos, destaca-se o delineamento de base populacional, com baixa proporção de perdas e recusas, além do rigor metodológico empregado no planejamento e coleta de dados.

\section{CONCLUSÃO}

Encontrou-se uma elevada prevalência de idosos em convívio com animais de estimação, contribuindo para o conhecimento desse desfecho numa população de idosos, sendo um dos únicos estudos de base populacional que avalia essa temática. Contudo mais estudos são necessários para melhor compreender como a convivência com os animais se reflete em melhorias de saúde para a população idosa.

\section{REFERÊNCIAS}

1. Bispo NNC, Furini TF, Fidelis TAS, Falossi LC, Molari M, Costa VSP. Envelhecimento bem-sucedido na perspectiva de pessoas idosas. In: Atas do 8o Congresso Ibero-Americano em Investigação Qualitativa [Internet]; 2019 jul 16-19 [acesso em 2020 jul. 25]; Lisboa, Portugal. p. 1303-11. Disponível em: https://proceedings.ciaiq.org/index.php/CIAIQ2019/article/view/2277/2198

2. Machado WD, Gomes DF, Freitas CASL, Brito MCC, Moreira ACA. Idosos com doenças crônicas não transmissíveis: um estudo em grupos de convivência. ReonFacema [Internet]. 2017 abr-jun [acesso em 2020 jul. 25]; 3(2): 444-51. Disponível em: http://www.facema.edu.br/ojs/index.php/ ReOnFacema/article/view/194/106

3. Silva AR, Sgnaolin V, Nogueira EL, Loureiro F, Engroff P, Gomes I. Doenças crônicas não transmissíveis e fatores sociodemográficos associados a sintomas de depressão em idosos. J. Bras. Psiquiatr [Internet]. 2017 mar; 66(1): 45-51. doi: https://doi.org/10.159o/o047-2085000000149

4. Meirelles JML, Fischer ML. O animal de estimação como membro da família: repercussões sociais, éticas e jurídicas. In: Anais do $5^{\circ}$ Congresso Mundial de Bioética e Direitos Animais [Internet]; 2016 out 26-28; Curitiba. Salvador: Fundação Orlando Gomes; 2017. p. 97-110. [acesso em 2020 jul 18]. Disponível em: https://wp.ufpel.edu.br/direitosdosanimais/files/2017/o2/Anais-dos-Congressos-de-Bio\% $33 \%$ Atica-e-Direito-Animal-I.pdf?file=2017/o2/Anais-dos-Congressos-de-Bio\%$\mathrm{C}_{3} \%$ Agtica-e-Direito-Animal-I.pdf

5. Costa EC, Jorge MSB, Saraiva ERA, Coutinho MPL. Aspectos psicossociais da convivência de idosas com animais de estimação: uma interação social alternativa. Psicol. teor. prat [Internet]. 2009 [acesso em 2016 jul 2o]; 11(3): 2-15 Disponível em: http://pepsic.bvsalud.org/scielo.php?script=sci_arttext\&pid=S1516-36872009000300002

6. Heiden J, Santos W. Benefícios psicológicos da convivência com animais de estimação para idosos. Ágora: rev. divulg. cient [Internet]. 2012 abr [acesso em 2020 jul 25]; 16(2esp): 487-96. Disponível em: http://www.periodicos.unc.br/index.php/agora/article/view/138 
7. Stumm, KE, Alves CN, de Medeiros PA, Ressel LB. Terapia assistida por animais como facilitadora no cuidado a mulheres idosas institucionalizadas. Rev Enferm UFSM [Internet]. 2012 jan.-abr [acesso em 2020 jul 25]; 2(1): 205-12. Disponível em: https://periodicos.ufsm.br/reufsm/article/ view/2616/3145

8. Faraco CB. Interação humano-animal. Ciênc. Vet. Tróp [Internet]. 2008 abr [acesso em 2020 jul 26]; 11(1): 31-5. Disponível em: http://www.rcvt.org.br/suplemento11/31-35.pdf

9. Fischer ML, Zanatta, AA, Adami ER. Um olhar da bioética para a zooterapia. Rev. Latinoam. Bioet [Internet]. 2016; 16(1): 174-97. doi: http://dx.doi.org/10.18359/rlbi.146o

10. Lima AS, Souza MB. Os benefícios apresentados na utilização da terapia assistida por animais: revisão de literatura. Rev. Saú. e Desenv [Internet]. 2018 [acesso em 2020 jul 20]; 12(10): 224-41. Disponível em: https://www.uninter.com/revistasaude/index.php/saudeDesenvolvimento/article/ view/880

11. Cechetti F, Pagnussat AS, Marin KE, Bertuol P, Todero FZ, Ballardim SAO. Terapia Assistida por Animais como recurso fisioterapêutico para idosos institucionalizados. Sci Med [Internet]. 2016 [acesso em 2020 jul 25]; 26(3): ID23686. Disponível em: https://revistaseletronicas.pucrs.br/ojs/ index.php/scientiamedica/article/view/23686/14871

12. Brasil, Ministério da Saúde. Banco de dados do Sistema Único de Saúde-DATASUS. [Internet] [acesso em 2020 jul 30]. Disponível em: http://tabnet.datasus.gov.br/cgi/tabcgi.exe?ibge/cnv/popRS.def

13. Sheikh JI, Yesavage JA. Geriatric depression scale (GDS): recent evidence and development of a shorter version. Clin Gerontol [Internet]. 1986; 5(1-2): 165-73. doi: /10.130o/Jo18vo5no1_o9

14. Giumelli RD, Santos MCP. Convivência com animais de estimação: um estudo fenomenológico. Rev. abordagem gestalt [Internet]. 2016 [acesso em 2020 jul 20]; 22(1): 49-58. Disponível em: http://pepsic.bvsalud.org/pdf/rag/v22n1/v22n1ao7.pdf

15. Alves L, Steyer S. Interação humano-animal. Perspec. em Psico [Internet]. 2019 [acesso em 2020 jul 20]; 23(2): 124-42. Disponível em: http://www.seer.ufu.br/index.php/perspectivasempsicologia/ article/view/52223

16. Santos RCB, Moura KB, Sousa E, de Oliveira RA, Soares BC, de Oliveira Melo W. Interação homem-animal de companhia no município de Paragominas, sudeste do Pará. Acta Vet Brasil [Internet]. 2016; 10(1): 55-62. doi: 10.21708/avb.2016.10.1.5478

17. Garcia DO, Wertheim BC, Manson JE, Chlebowski RT, Volpe SL, Howard BV et al. Relationships between dog owner ship and physical activity in postmenopausal women. Prev. Med [Internet]. 2015; 70: 33-8. doi: 10.1016/j.ypmed.2014.10.030 
18. Pongelupe TY, Ferraz FHA, Raposo JA, Martin DW, Santos AS, Pereira CAD. Atividade assistida por animais em integrantes do Grupo de Bem Estar e Qualidade de Vida do Idoso de uma universidade - TAA - parte II. Saúde Coletiva [Internet]. 2009 [acesso em 2020 jul 30]; 6(33): 212-16. Disponível em: https://www.redalyc.org/articulo.oa?id=84212107005

19. Potter P, Perry A. Fundamentos de enfermagem. 8a ed. Rio de Janeiro: Guanabara Koogan; 2014.

20. Souto CCL, Silva RCF, Porto CM, Zimmermann RD, Costa MLG. Convívio domiciliar de idosos com cães de estimação. Estud. Interdiscipl. Envelhec [Internet]. 2019 [acesso em 2020 jul 21]; 24(3): 4-21. Disponível em: https://www.seer.ufrgs.br/RevEnvelhecer/article/viewFile/93839/57059

21. Instituto Brasileiro de Geografia e Estatística. [Internet]. [acesso em 2020 nov 17]. Disponível em: https://cidades.ibge.gov.br/brasil/rs/bage/panorama 